\title{
Electrodynamics of Balanced Charges
}

\author{
Anatoli Babin · Alexander Figotin
}

Received: 20 April 2010 / Accepted: 18 August 2010 / Published online: 2 September 2010

(C) The Author(s) 2010. This article is published with open access at Springerlink.com

\begin{abstract}
We introduce here a new "neoclassical" electromagnetic (EM) theory in which elementary charges are represented by wave functions and individual EM fields to account for their EM interactions. We call so defined charges balanced or "b-charges". We construct the EM theory of b-charges (BEM) based on a relativistic field Lagrangian and show that: (i) the elementary EM fields satisfy the Maxwell equations; (ii) the Newton equations with the Lorentz forces hold approximately when b-charges are well separated and move with non-relativistic velocities. When the BEM theory is applied to atomic scales it yields a hydrogen atom model with a frequency spectrum matching the Schrodinger model with desired accuracy. An important feature of the theory is a mechanism of elementary EM energy absorption established for retarded potentials.
\end{abstract}

Keywords Electromagnetic theory · Lagrangian · Wave-corpuscle · Elementary absorption

\section{Introduction}

It is well recognized that the classical electromagnetic (CEM) theory formulated in the form of Maxwell-Lorentz equations provides an excellent description of electromagnetic phenomena at the macroscopic length scales. It is also well known that CEM theory is inadequate in explaining electromagnetic (EM) phenomena at the atomic scales including spectroscopic data of the hydrogen atom (HA). We develop here a "neo-classical" EM theory which accounts for all classical EM phenomena

\footnotetext{
A. Babin · A. Figotin $(\bowtie)$

Department of Mathematics, The University of California at Irvine, Irvine, CA 92697, USA

e-mail: afigotin@uci.edu
}

\section{A. Babin \\ e-mail: ababine@uci.edu}


at the macroscopic scales as well at least some EM phenomena at the atomic scale including the HA spectral lines. When attempting to change the CEM theory we want: (i) to stay on the solid ground of the Lagrangian mechanics and the relativity principle; (ii) to recover in this new EM theory all well established experimental facts described by the CEM theory. Before we proceed with the new developments, let us briefly recall the CEM theory fundamentals. The EM field associated with a prescribed current in vacuum is described by the Maxwell equations

$$
\begin{aligned}
\frac{1}{\mathrm{c}} \partial_{t} \mathbf{B}+\nabla \times \mathbf{E}=\mathbf{0}, & \nabla \cdot \mathbf{B}=0, \\
\frac{1}{\mathrm{c}} \partial_{t} \mathbf{E}-\nabla \times \mathbf{B}=-\frac{4 \pi}{\mathrm{c}} \mathbf{J}, & \nabla \cdot \mathbf{E}=4 \pi \rho,
\end{aligned}
$$

where $\mathbf{E}, \mathbf{B}, \rho(t, x), \mathbf{J}(t, x)$ are respectively the electric field, the magnetic induction, prescribed charge and current densities. In particular, for point charges

$$
\rho=\sum_{\ell=1}^{N} q^{\ell} \delta\left(\mathbf{x}-\mathbf{r}^{\ell}(t)\right), \quad \mathbf{J}=\sum_{\ell=1}^{N} q^{\ell} \delta\left(\mathbf{x}-\mathbf{r}^{\ell}(t)\right) \mathbf{v}^{\ell}(t),
$$

where $q^{\ell}$ is the value of the $\ell$-th point charge, $\mathbf{r}^{\ell}$ and $\mathbf{v}^{\ell}(t)=\frac{\mathrm{d} \mathbf{r}^{\ell}}{\mathrm{d} t}$ are respectively its position and velocity, and $\delta$ is the Dirac delta-function. For a given EM field the motion of every point charge in the EM field is determined by

$$
\frac{\mathrm{d}}{\mathrm{d} t}\left[m^{\ell} \mathbf{v}^{\ell}(t)\right]=q^{\ell}\left[\mathbf{E}\left(t, \mathbf{r}^{\ell}(t)\right)+\frac{1}{\mathrm{c}} \mathbf{v}^{\ell}(t) \times \mathbf{B}\left(t, \mathbf{r}^{\ell}(t)\right)\right],
$$

where $m^{\ell}$ is the $\ell$-th point charge mass and the right hand side of (4) is the Lorentz force. The CEM theory treats three types of problems: (i) studies of EM fields for prescribed charge and current densities; (ii) the motion of charges in a prescribed external field; (iii) interaction of charges and their EM fields. The classical MaxwellLorentz system though very successful in describing many EM phenomena has well known problems. One of those is the lack of an "elementary process of absorption" pointed out by A. Einstein in [6]: "According to the prevailing theory an oscillating ion produces an outwardly propagated spherical wave. The opposite process does not exist as an elementary process. It is true that the inwardly propagated spherical wave is mathematically possible; however its approximate realization requires an enormous amount of emitting elementary structures. Thus, the elementary process of light radiation as such does not possess the character of reversibility. Here, I believe, our wave theory is off the mark."

The proposed here EM theory is based on a new concept for elementary charge which we call a balanced charge or b-charge, and we refer to the theory as to BEM theory. One of its key elements is a concept of an elementary EM field assigned to every $b$-charge in contrast with a single EM field in the CEM theory. A single b-charge is described by a pair $\left(\psi, A^{\mu}\right)$, where $\psi$ is its wave function and $A^{\mu}=$ $(\varphi, \mathbf{A})$ is its 4 -vector elementary potential. So, a b-charge is a field $\left(\psi, A^{\mu}\right)$ over 4-dimensional space-time continuum, in which the wave function $\psi$ describes the 
charge distribution and the elementary potential $A^{\mu}$ mediates its EM interactions with all other b-charges. Importantly, (i) all internal forces of a b-charge are exclusively of non-electromagnetic origin; (ii) every b-charge is a source of its elementary EM field which represents force exerted by this charge on any other b-charge but not upon itself. The later allows to view a single b-charge as truly elementary one with respect to the electromagnetic interactions. The proposed here theory does have an elementary process of absorption, we call it "negative radiation".

An idea to introduce an extended charge instead of the point one is not new, and the most known models for it are the Abraham rigid charge model and the Lorentz relativistically covariant model. These models are studied and advanced in many papers, see [9, Sect. 16], [10, 13], [14, Sect. 2, 6], [16, 17, 22]. In contrast to those models, here and in $[1,2]$ we do not prescribe to an elementary charge a certain geometry, and do not prescribe Newtonian dynamics for its center, but instead the elementary charge has a wave function governed by a nonlinear Klein-Gordon or a nonlinear Schrödinger equation in the relativistic and nonrelativistic cases respectively. An idea to eliminate self-interaction is also, of course, not new. The latest to our best knowledge attempt to have this feature in the electrodynamics is due J. Wheeler and R. Feynman [20, 21], but the EM theory proposed here is very different from it.

The BEM theory is constructed based on a relativistic Lagrangian with the following properties: (i) b-charges interact only through their elementary EM fields; (ii) the field equations for the elementary EM fields are exactly the Maxwell equations with proper currents; (iii) a free charge moves uniformly preserving up to the Lorentz contraction its shape; (iv) the Newton equations with the Lorentz forces hold approximately when charges are well separated and move with non-relativistic velocities. Since an overwhelming number of EM phenomena are explained within the CEM theory by the Maxwell equations and the Lorentz forces the BEM theory is equally successful in explaining the same phenomena.

A system of $N$ elementary charges in the BEM theory is modeled by $N$ pairs $\left(\psi^{\ell}, A^{\ell \mu}\right), 1 \leq \ell \leq N$. The classical (total) $\mathrm{EM}$ field $A^{\mu}=(\varphi, \mathbf{A})$ is recovered in this theory as the sum of all elementary EM fields, namely

$$
\varphi=\sum_{\ell=1}^{N} \varphi^{\ell}, \quad \mathbf{A}=\sum_{\ell=1}^{N} \mathbf{A}^{\ell}
$$

but, importantly, this total field is not an independent entity. Notice then that since in the BEM theory there is no EM self-interaction the action on $\ell$-th charge by EM fields of other charges is described by a field $A_{\neq \ell}^{\mu}=\left(\varphi \neq \ell, \mathbf{A}_{\neq \ell}\right)$ which is the total field $(\varphi, \mathbf{A})$ "balanced" by the removal from it the self-interaction, namely

$$
\varphi_{\neq \ell}=\sum_{\ell^{\prime} \neq \ell} \varphi^{\ell^{\prime}}, \quad \mathbf{A}_{\neq \ell}=\sum_{\ell^{\prime} \neq \ell} \mathbf{A}^{\ell} .
$$

Use of EM fields similar to ones in (6) is, of course, not a discovery and they can be found in many textbooks, but the BEM theory goes further and consistently removes the elementary EM self-actions for the CEM Lagrangian and consequently the elementary self-energies from the classical EM energy-momentum tensor. Only with this 
removal of the elementary self-actions from the classical EM Lagrangian one gets the field equations in the form of the elementary Maxwell equations $\partial_{\mu} F^{\ell \mu \nu}=\frac{4 \pi}{\mathrm{c}} J^{\ell \nu}$ for the elementary EM fields $F^{\ell \mu \nu}$ with the elementary conserved 4-currents $J^{\ell v}$. In the BEM theory the EM energy is the energy of EM interaction of pairs of b-charges and consequently it is naturally partitioned between the pairs of charges. We show that for every pair of b-charges their EM interaction energy satisfies an elementary energymomentum conservation law governed by the relevant Lorentz force densities.

We also show that the CEM theory is a limit of the BEM theory at the macroscopic scale, but the differences between the CEM and BEM theories become more pronounced for systems with fewer b-charges. The BEM theory predictions can significantly deviate from those of the CEM theory in the following situations: (i) there are just a few b-charges which are in close proximity; (ii) there is a large but highly coherent system of b-charges similar to those collective, coherent systems (superconducting ring, laser and more) described by C. Mead in [11, p. 5].

\section{Relativistic Theory}

\subsection{Lagrangian, Field Equations, Currents and Energy-momentum Tensors}

Let us consider a system of elementary charges $\left(\psi^{\ell}, A^{\ell \mu}\right), 1 \leq \ell \leq N$. It is a key element of the construction that the $\ell$-th charge potential $A^{\ell \mu}$ and its EM field $F^{\ell \mu \nu}=\partial^{\mu} A^{\ell v}-\partial^{\nu} A^{\ell \mu}$ completely account for its action upon all other charges $\ell^{\prime} \neq \ell$. Consequently, the action upon $\ell$-th charge by all other charges is described by $\ell$-th exterior potential $A_{\neq}^{\ell \mu}$ and its EM field $F_{\neq}^{\ell \mu \nu}$ defined by

$$
A_{\neq}^{\ell \mu}=\sum_{\ell^{\prime} \neq \ell} A^{\ell^{\prime} \mu}, \quad A_{\neq}^{\ell \mu}=\left(\varphi_{\neq}^{\ell}, \mathbf{A}_{\neq}^{\ell}\right), \quad F_{\neq}^{\ell \mu \nu}=\sum_{\ell^{\prime} \neq \ell} F^{\ell^{\prime} \mu \nu} .
$$

We also introduce the total potential $\mathcal{A}^{\mu}$ and the corresponding total EM field $\mathcal{F}^{\mu \nu}$ by the following formulas

$$
\mathcal{A}^{\mu}=\sum_{\ell=1}^{N} A^{\ell \mu}, \quad \mathcal{F}^{\mu \nu}=\sum_{\ell=1}^{N} F^{\ell \mu \nu} .
$$

We furnish then the system of $N$ b-charges with the following Lagrangian

$$
\mathcal{L}\left(\left\{\psi^{\ell}, \psi_{; \mu}^{\ell}\right\},\left\{\psi^{\ell *}, \psi_{; \mu}^{\ell *}\right\}, A^{\ell \mu}\right)=\sum_{\ell=1}^{N} L^{\ell}\left(\psi^{\ell}, \psi_{; \mu}^{\ell}, \psi^{\ell *}, \psi_{; \mu}^{\ell *}\right)+\mathcal{L}_{\mathrm{BEM}}
$$

where $\psi^{*}$ is complex conjugate to $\psi$, the EM part of the Lagrangian is

$$
\mathcal{L}_{\mathrm{BEM}}=-\frac{1}{16 \pi} \sum_{\left\{\ell, \ell^{\prime}: \ell^{\prime} \neq \ell\right\}} F^{\ell \mu \nu} F_{\mu \nu}^{\ell^{\prime}}=-\frac{1}{16 \pi} \sum_{\ell=1}^{N} F^{\ell \mu \nu} F_{\neq \mu \nu}^{\ell} .
$$


The EM part $\mathcal{L}_{\mathrm{BEM}}$ of the Lagrangian $\mathcal{L}$ is obtained by the removal from the classical EM Lagrangian $\mathcal{L}_{\text {CEM }}$ all self-interaction contributions of the elementary EM fields. The "bare" charge Lagrangians $L^{\ell}$ are nonlinear Klein-Gordon Lagrangians defined by exactly same expressions as in $[1,2]$, namely

$$
L^{\ell}\left(\psi^{\ell}, \psi_{; \mu}^{\ell}, \psi^{\ell *}, \psi_{; \mu}^{\ell *}\right)=\frac{\chi^{2}}{2 m^{\ell}}\left\{\psi_{; \mu}^{\ell *} \psi^{\ell ; \mu}-\kappa^{\ell 2} \psi^{\ell *} \psi^{\ell}-G^{\ell}\left(\psi^{\ell *} \psi^{\ell}\right)\right\},
$$

where (i) $G^{\ell}$ is a nonlinear self-interaction function of the $\ell$-th charge described below; (ii) $m^{\ell}>0$ is the charge mass parameter; (iii) $q^{\ell}$ is the value of the charge; (iv) $\chi>0$ is a parameter similar to the Planck constant $\hbar=\frac{h}{2 \pi}$ and $\kappa^{\ell}=\frac{m^{\ell} \mathrm{c}}{\chi}$, $\omega^{\ell}=\frac{m^{\ell} \mathrm{c}^{2}}{\chi}$, (v) the covariant derivatives are defined by the formulas $\psi_{; \mu}^{\ell}=\partial^{\mu} \psi^{\ell}+$ $\frac{\mathrm{i} q^{\ell}}{\chi \mathrm{c}} A_{\neq}^{\ell \mu} \psi^{\ell}$. The Euler-Lagrange field equations for the above Lagrangian $\mathcal{L}$ are (i) elementary Klein-Gordon equations

$$
-\frac{1}{\mathrm{c}^{2}} \tilde{\partial}_{t}^{\ell} \tilde{\partial}_{t}^{\ell} \psi^{\ell}+\tilde{\nabla}^{\ell 2} \psi^{\ell}-G^{\ell \prime}\left(\psi^{\ell *} \psi^{\ell}\right) \psi^{\ell}-\kappa_{0}^{2} \psi^{\ell}=0, \quad \ell=1, \ldots, N
$$

where

$$
\tilde{\nabla}^{\ell}=\nabla-\frac{\mathrm{i} q^{\ell}}{\chi c} \mathbf{A}_{\neq}^{\ell}, \quad \tilde{\partial}_{t}^{\ell}=\partial_{t}+\frac{\mathrm{i} q^{\ell}}{\chi} \varphi_{\neq}^{\ell},
$$

together with the conjugate equation for $\psi^{* \ell}$ and (ii) the Maxwell equations for the elementary EM fields with the corresponding elementary currents

$$
\partial_{\mu} F^{\ell \mu \nu}=\frac{4 \pi}{\mathrm{c}} J^{\ell v}, \quad \ell=1, \ldots, N
$$

where $J^{\ell v}=\left(\mathrm{c} \rho^{\ell}, \mathbf{J}^{\ell}\right)$ is the $\ell$-th elementary current presented as follows:

$$
\begin{aligned}
\rho^{\ell} & =-\frac{q^{\ell}}{m^{\ell} \mathrm{c}^{2}}\left(\chi \operatorname{Im} \frac{\partial_{t} \psi^{\ell}}{\psi^{\ell}}+q^{\ell} \varphi_{\neq}^{\ell}\right)\left|\psi^{\ell}\right|^{2}, \\
\mathbf{J}^{\ell} & =\frac{q^{\ell}}{m^{\ell}}\left(\chi \operatorname{Im} \frac{\nabla \psi^{\ell}}{\psi^{\ell}}-\frac{q^{\ell}}{\mathrm{c}} \mathbf{A}_{\neq}^{\ell}\right)\left|\psi^{\ell}\right|^{2} .
\end{aligned}
$$

One can see from the elementary wave equation (12) that the $\ell$-th charge is driven by its exterior potential $A_{\neq}^{\ell v}$ indicating that there is no EM self-interaction. The summation of the Maxwell equations (13) for the elementary EM fields readily implies that the total EM field $\mathcal{F}^{\mu \nu}$ defined by (7) also satisfies the Maxwell equations with the total currents being the sum of all the elementary currents as in the CEM theory. One can interpret the total EM field $\mathcal{F}^{\mu \nu}$ as one acting upon a $(N+1)$-th test charge which is so small that its action onto the system of $N$ charges can be neglected.

The system Lagrangian $\mathcal{L}$ defined by (9)-(11) is manifestly Lorentz and gauge invariant with respect to the gauge transformations of the first kind [12, 19]. The gauge invariance via the Noether's theorem allows to introduce as usual elementary conserved currents which coincide with the currents $J^{\ell v}$ defined above, see [1,2] for 
details. The currents satisfy for every $\ell$ the conservation law

$$
\partial_{v} J^{\ell v}=0 \quad \text { or } \quad \partial_{t} \rho^{\ell}+\nabla \cdot \mathbf{J}^{\ell}=0 .
$$

In what follows we also use a vector form of field equations (13) namely the Maxwell equations for all elementary EM potentials $\varphi^{\ell}, \mathbf{A}^{\ell}$

$$
\begin{aligned}
& \nabla \cdot\left(\frac{1}{\mathrm{c}} \partial_{t} \mathbf{A}^{\ell}+\nabla \varphi^{\ell}\right)=-4 \pi \rho^{\ell}, \quad \ell=1, \ldots, N, \\
& \nabla \times\left(\nabla \times \mathbf{A}^{\ell}\right)+\frac{1}{\mathrm{c}} \partial_{t}\left(\frac{1}{\mathrm{c}} \partial_{t} \mathbf{A}^{\ell}+\nabla \varphi^{\ell}\right)=\frac{4 \pi \mathbf{J}^{\ell}}{\mathrm{c}},
\end{aligned}
$$

where $\rho^{\ell}$ and $\mathbf{J}^{\ell}$ are defined by (14). Equations (16)-(17) can be written in the form (1), (2) if we set as usual

$$
\mathbf{E}=-\nabla \varphi-\frac{1}{\mathrm{c}} \partial_{t} \mathbf{A}, \quad \mathbf{B}=\nabla \times \mathbf{A} .
$$

If the Lorentz gauge is imposed, (16)-(17) turn into the wave equations for $\psi^{\ell}, \mathbf{A}^{\ell}$. Importantly, as in the wave-corpuscle mechanics (WCM) introduced and studied in $[1,2]$, the nonlinearities $G^{\ell}$ are determined based on the single $\ell$-th charge equation (12), (16), (17) where we set $N=1$ and consequently $A_{\neq}^{\ell \mu}=0$. The rest state of the $b$-charge is defined to be of the form (as in the WCM, $[1,2]$ )

$$
\psi^{\ell}(t, \mathbf{x})=\mathrm{e}^{-\mathrm{i} \omega_{0} t} \dot{\psi}(|\mathbf{x}|), \quad \mathbf{A}^{\ell}(t, \mathbf{x})=\mathbf{0}, \quad \varphi^{\ell}(t, \mathbf{x})=\stackrel{\circ}{\varphi}(|\mathbf{x}|)
$$

where $\omega_{0}=m^{\ell} \mathrm{c}^{2} / \chi, \dot{\psi}$ and $\stackrel{\circ}{\varphi}$ are real-valued radial functions. Substituting the $\psi$, $\varphi$ and A defined by the relations (19) into (12) we obtain the following rest charge equations:

$$
-\nabla^{2} \dot{\psi}+G^{\prime}\left(|\stackrel{\circ}{\psi}|^{2}\right) \stackrel{\circ}{\psi}=0, \quad-\nabla^{2} \stackrel{\circ}{\varphi}=4 \pi|\dot{\psi}|^{2} .
$$

The quantity $\dot{\psi}$ is fundamental for our theory and we call it form factor. Equation (20) establishes an explicit relation between the form factor $\dot{\psi}$ and the self-interaction nonlinearity $G$. If the form factor ${ }^{\prime}(r)$ is given and it is a nonnegative, monotonically decaying and sufficiently smooth function of $r \geq 0$, we can find from the equilibrium equation (20) the self-interaction nonlinearity $G$ which exactly produces this factor. We pick the form factor $\dot{\psi}$ considering it as the model parameter and then the nonlinear self interaction function $G$ is determined based on the charge equilibrium equation (20). To explicitly integrate the size of the resting b-charge into its model we introduce a size parameter $a>0$ into $G=G_{a}$ as follows:

$$
G_{a}^{\prime}(s)=a^{-2} G_{1}^{\prime}\left(a^{3} s\right), \quad \text { where } G^{\prime}(s)=\partial_{s} G(s) .
$$

In Sect. 3.1 we give examples of the nonlinearity $G$ and discuss its properties.

Since our theory is relativistic and gauge invariant, it possesses usual conservation laws. Applying with minor modifications a general method used in [1,2] one can find for the Lagrangian $\mathcal{L}$ defined by (9)-(11) the energy-momentum tensors (EnMT) and 
corresponding conservation laws in which the Lorentz force densities naturally arise. A general source of conservation laws in Lagrangian theories is Noether's theorem, [7, 13.7], yielding them canonically from Lagrangian symmetries. The elementary charge and energy-momentum conservation laws hold for every single charge assuring its individuality. The symmetric EnMT $\mathcal{T}^{\mu \nu}$ of the system Lagrangian $\mathcal{L}$ defined by (10)-(11) is represented by

$$
\mathcal{T}^{\mu \nu}=\sum_{\ell=1}^{N} T^{\ell \mu \nu}+\Xi^{\mu \nu}, \quad \Xi^{\mu \nu}=\sum_{\ell^{\prime} \neq \ell} \Xi^{\ell \ell^{\prime} \mu \nu},
$$

where (i) $\Xi^{\ell \ell^{\prime} \mu \nu}$ is given by the standard expression for EnMT of EM fields, see [9, Sect. 12.10]; (ii) the individual EnMT $T^{\ell \mu \nu}$ of the bare $\ell$-th charge are defined by standard expressions for Klein-Gordon EnMT, as in [1, 2] with obvious modifications. Note that the total symmetric classical EnMT is obtained similarly to $\Xi^{\mu \nu}$ in (22) but with the summation over all $\ell, \ell^{\prime}$ without restriction $\ell^{\prime} \neq \ell$. This leads to some differences which are negligible for most of large systems but in some special cases may become noticeable as we show below.

The total symmetric EnMT $\mathcal{T}^{\mu \nu}$ defined by the expression (22) satisfies the conservation law $\partial_{\mu} \mathcal{T}^{\mu \nu}=0$. The energy density $w^{\ell \ell^{\prime}}$ and the Poynting vectors $\mathbf{S}^{\ell \ell^{\prime}}$ for ordered pair $\ell \ell^{\prime}$ in vector notation are

$$
w^{\ell \ell^{\prime}}=\frac{1}{8 \pi}\left(\mathbf{E}^{\ell} \cdot \mathbf{E}^{\ell^{\prime}}+\mathbf{B}^{\ell} \cdot \mathbf{B}^{\ell^{\prime}}\right), \quad \mathbf{S}^{\ell \ell^{\prime}}=\frac{\mathbf{c} \mathbf{E}^{\ell} \times \mathbf{B}^{\ell^{\prime}}}{4 \pi} .
$$

The elementary EnMT satisfy the following elementary conservation laws

$$
\partial_{\mu}\left(\Xi^{\ell \ell^{\prime} \mu \nu}+\Xi^{\ell^{\prime} \ell \mu \nu}\right)=-\frac{1}{\mathrm{c}}\left(J_{\xi}^{\ell} F^{\ell^{\prime} \nu \xi}+J_{\xi}^{\ell^{\prime}} F^{\ell \nu \xi}\right)
$$

with the right-hand side being the negative of the sum of the corresponding Lorentz force densities. The vector form of the elementary EM energy conservation (24) can be easily derived from the classical Poynting theorem. The individual EnMT satisfy the following elementary conservation laws

$$
\partial_{\mu} T^{\ell \mu \nu}=\frac{1}{\mathrm{c}} J_{\xi}^{\ell} \sum_{\ell^{\prime} \neq \ell} F^{\ell^{\prime} \nu \xi}=\frac{1}{\mathrm{c}} J_{\xi}^{\ell} F_{\neq}^{\ell \nu \xi} .
$$

Observe that the right-hand side of the above equality is the Lorentz force density and the same expressions with the minus sign arise in the conservations laws (24) for the EM fields.

\subsection{Elementary EM Fields for Prescribed Elementary Currents}

To study the properties of the elementary EM fields for balanced changes and the energy-momentum transfer in the space-time it is instructive to consider a situation of prescribed currents for b-charges similarly to the same for the case of the CEM theory. To do that we consider the Maxwell equations (16), (17) for elementary EM 
field for every $\ell$ and assume that the currents $\mathbf{J}$ and densities $\rho$ in the equations are known (prescribed) functions of $(t, \mathbf{x})$. The solutions to these equations are derived based on standard well known methods. Importantly, in what follows we consider only EM fields based on the retarded/causal Green functions. The advanced Green functions and the fields are important in some EM theories, see [23] and references therein. It would be interesting to explore their possible role in our theory but such a study is beyond the scope of this paper.

Let us assume for simplicity all prescribed elementary currents to be in the form of ideal electric dipoles. Recall that an ideal electric dipole source concentrated at a point $\mathbf{x}_{0}$ is defined as follows, [18, (7.151), App. 8]:

$$
\mathbf{J}(t, \mathbf{x})=\dot{\mathbf{p}}(t) \delta\left(\mathbf{x}-\mathbf{x}_{0}\right), \quad \rho(t, \mathbf{x})=-\mathbf{p}(t) \cdot \nabla \delta\left(\mathbf{x}-\mathbf{x}_{0}\right),
$$

where $\delta\left(\mathbf{x}-\mathbf{x}_{0}\right)$ is the Dirac delta-function, $\dot{\mathbf{p}}(t)=\partial_{t} \mathbf{p}(t)$. In a simpler case when the dipole function $\mathbf{p}(t)=\mathbf{p}^{\ell}(t)=\mathbf{p}_{\omega} \mathrm{e}^{-\mathrm{i} \omega t}$ is time harmonic in the radiation zone $\frac{\omega}{\mathrm{c}}|\mathbf{R}| \gg 1$ we use the asymptotic expressions for $\mathbf{E}(t, \mathbf{x})$ and $\mathbf{B}(t, \mathbf{x})$ for the radiation field from $[9,9.2],[8,11.1 .4]$. As a result for a pair of charges with indices $\ell, \ell^{\prime}$ we obtain the following formulas for the interaction energy flux $\mathbf{S}^{\ell \ell^{\prime}}$ :

$$
\mathbf{S}^{\ell \ell^{\prime}}=\frac{\mathbf{c} \mathbf{E}^{\ell} \times \mathbf{B}^{\ell^{\prime}}}{4 \pi}=\frac{\left[\left(\ddot{\mathbf{p}}^{\ell}\left(t_{0}\right) \times \hat{\mathbf{R}}\right) \cdot\left(\ddot{\mathbf{p}}^{\ell^{\prime}}\left(t_{0}\right) \times \hat{\mathbf{R}}\right)\right] \hat{\mathbf{R}}}{4 \pi \mathrm{c}^{3}|\mathbf{R}|^{2}},
$$

where

$$
\mathbf{R}=\mathbf{x}-\mathbf{x}_{0}, \quad \hat{\mathbf{R}}=\mathbf{R} /|\mathbf{R}|, \quad t_{0}=t-|\mathbf{R}| / \mathrm{c} .
$$

Then the corresponding power $P^{\ell \ell^{\prime}}$ radiated through a sphere of a large radius centered at the origin is

$$
P^{\ell \ell^{\prime}}=\int_{|\mathbf{x}|=|\mathbf{R}|} \mathbf{S}^{\ell \ell^{\prime}} \mathrm{d} \sigma=\frac{2}{3 \mathrm{c}^{3}} \ddot{\mathbf{p}}^{\ell}\left(t_{0}\right) \cdot \ddot{\mathbf{p}}^{\ell^{\prime}}\left(t_{0}\right) .
$$

Let us assume now that all the dipole functions $\mathbf{p}^{\ell}(t)$ depend on time $t$ almost periodically, [5]. Then the representation (27) implies the following formulas for the time-averaged radiated powers

$$
\left\langle P^{\ell \ell^{\prime}}+P^{\ell^{\prime} \ell}\right\rangle=\frac{4\left\langle\ddot{\mathbf{p}}^{\ell} \cdot \ddot{\mathbf{p}}^{\ell^{\prime}}\right\rangle}{3 \mathrm{c}^{3}}=\sum_{\omega \in \Lambda_{\mathbf{p}} \cap \cap \Lambda_{\mathbf{p}^{\ell^{\prime}}}} \frac{2 \omega^{4} \operatorname{Re}\left(\mathbf{p}_{\omega}^{\ell} \cdot \mathbf{p}_{\omega}^{\ell^{\prime} *}\right)}{3 \mathrm{c}^{3}},
$$

where $\Lambda_{\mathbf{p}^{\ell}}$ and $\Lambda_{\mathbf{p}^{\ell^{\prime}}}$ are respectively the frequency spectra of $\mathbf{p}^{\ell}(t)$ and $\mathbf{p}^{\ell^{\prime}}(t),\langle\cdot\rangle$ denotes time averaging. It readily follows from (28) that the time-averaged radiated power of two dipoles $\left\langle P^{\ell \ell^{\prime}}+P^{\ell^{\prime} \ell}\right\rangle$ can take any real value: negative, zero or positive. In particular, the relation (28) implies that if both the $\ell$-th and $\ell^{\prime}$-th $b$-charges are monochromatic and of different frequencies then the time-averaged radiated power is exactly zero or in other words there is no radiation. The formulas (28) also readily imply

$$
\left\langle P^{\ell \ell^{\prime}}+P^{\ell^{\prime} \ell}\right\rangle=\frac{ \pm 2}{3 \mathrm{c}^{3}} \sum_{\omega \in \Lambda_{\mathbf{p}} \ell} \omega^{4}\left|\mathbf{p}_{\omega}^{\ell}\right|^{2} \quad \text { if } \mathbf{p}^{\ell^{\prime}}(t)= \pm \mathbf{p}^{\ell}(t) .
$$


Evidently the relation (29) with the minus sign describes a situation when for a given pair $\left(\ell, \ell^{\prime}\right)$ the time-averaged radiated power $\left\langle P^{\ell \ell^{\prime}}+P^{\ell^{\prime} \ell}\right\rangle$ is negative, that is the radiated energy propagates with the speed of light toward the source rather than away from it. Note that formula (29) describes the radiation of EM interaction energy between the two charges, and evidently it is not the same as the total EM radiation of the fields which act on test charges.

\subsection{Comparison with the Classical EM Theory}

Here we briefly discuss similarities and differences between BEM and CEM theories and show that the CEM theory is a limit case of the BEM theory. As a first example let us consider a system of $N>1 \mathrm{~b}$-charges described by one and the same dipole moment $\mathbf{p}(t)$. Then in view of (29) we have for the system the following time-average radiated power

$$
\left\langle P_{\mathrm{BEM}}\right\rangle=\sum_{\ell^{\prime} \neq \ell}\left\langle P^{\ell \ell^{\prime}}+P^{\ell^{\prime} \ell}\right\rangle=\frac{2 N(N-1)}{3 \mathrm{c}^{3}} \sum_{\omega \in \Lambda_{\mathbf{p}}} \omega^{4}\left|\mathbf{p}_{\omega}\right|^{2} .
$$

In the CEM theory a similar system of $N$ identical dipoles $\mathbf{p}$ has an effective dipole moment $N \mathbf{p}$. Substituting this number for $\mathbf{p}$ in the expression (29) we get

$$
\left\langle P_{\mathrm{CEM}}\right\rangle=\frac{2 N^{2}}{3 \mathrm{c}^{3}} \sum_{\omega \in \Lambda_{\mathbf{p}}} \omega^{4}\left|\mathbf{p}_{\omega}\right|^{2} .
$$

Relating representations (30) and (31) we readily obtain

$$
\left\langle P_{\mathrm{BEM}}\right\rangle=(1-1 / N)\left\langle P_{\mathrm{CEM}}\right\rangle .
$$

As a second example let us consider clusters of many tightly bound identical bcharges and label every cluster by index $\ell$. Namely, for every $\ell$ we introduce (i) $N_{\ell}$ b-charges having identical wave functions and elementary EM potentials with charges $q_{\mathrm{w}}^{\ell}=N_{\ell} q^{\ell}$ :

$$
\psi^{(\ell, s)}=\psi^{(\ell, s)}=\psi^{\ell}, \quad A^{(\ell, s) \mu}=A^{(\ell, s) \mu} \quad \text { where } 1 \leq \ell \leq N, 1 \leq s \leq N_{\ell},
$$

and (ii) their identical EM fields $F^{\ell \mu \nu}=F^{(\ell, s) \mu \nu}$ and currents $J^{\ell, v}=J^{(\ell, s) \nu}$. Then we compare these fields with the classical EM fields $\mathcal{F}^{\mu v}$ in (8) with the same currents, namely we compare electromagnetic parts of the Lagrangians in BEM and CEM theories. We have

$$
\mathcal{L}_{\mathrm{CEM}}=-\frac{1}{16 \pi} \mathcal{F}^{\mu \nu} \mathcal{F}_{\mu \nu}=-\frac{1}{16 \pi} \sum_{\ell^{\prime} \neq \ell} N_{\ell^{\prime}} N_{\ell} F^{\ell \mu \nu} F_{\mu \nu}^{\ell^{\prime}}-\frac{1}{16 \pi} \sum_{\ell=1}^{N} N_{\ell}^{2} F^{\ell \mu \nu} F_{\mu \nu}^{\ell},
$$

and (10) takes the form

$$
\mathcal{L}_{\mathrm{BEM}}=-\frac{1}{16 \pi} \sum_{\left\{(\ell, s),\left(\ell^{\prime}, s^{\prime}\right):\left(\ell^{\prime}, s^{\prime}\right) \neq(\ell, s)\right\}} F^{(\ell, s) \mu \nu} F_{\mu \nu}^{\left(\ell^{\prime}, s^{\prime}\right)}
$$




$$
=-\frac{1}{16 \pi} \sum_{\ell^{\prime} \neq \ell} N_{\ell^{\prime}} N_{\ell} F^{\ell \mu \nu} F_{\mu \nu}^{\ell^{\prime}}-\frac{1}{16 \pi} \sum_{\ell} N_{\ell}\left(N_{\ell}-1\right) F^{\ell \mu \nu} F_{\mu \nu}^{\ell} .
$$

The difference in both expressions can be attributed to interactions inside every cluster, in particular for classical theory $\mathcal{L}_{\mathrm{CEM} \ell}=N_{\ell}^{2} F^{\ell \mu \nu} F_{\mu \nu}^{\ell}$ compared with $\mathcal{L}_{\mathrm{BEM} \ell}=$ $N_{\ell}\left(N_{\ell}-1\right) F^{\ell \mu \nu} F_{\mu \nu}^{\ell}$ with the relative difference

$$
\mathcal{L}_{\mathrm{BEM} \ell} / \mathcal{L}_{\mathrm{CEM} \ell}-1=1 / N_{\ell}
$$

which evidently becomes small as number $N_{\ell}$ of charges in the cluster becomes large.

The differences between BEM and CEM theories can be already seen from expressions (32) and (34) if the numbers $N$ or $N_{\ell}$ of elementary charges are small. The fundamental origin of the differences is that in the BEM theory there is no single EM field as a reservoir of EM energy. Instead there are elementary EM potentials and all of the EM energy is the energy of interaction of pairs of the elementary b-charges. Importantly, the interactions between the charges cannot be exactly reduced to any single EM field, and this feature becomes more pronounced at atomic scales.

An important signature of the BEM theory is a mechanism of negative radiation for certain prescribed currents, i.e. a situation when the EM energy propagates with the speed of light toward the current source rather than away from it as it is shown in Sect. 2.2. This mechanism can conceivably work for a limited time in a system of several bound charges, such as an atom or a molecule, resulting in effective energy gain coming from matching energy loss of b-charges outside of this system. Notice that such energy transfer is accounted based on the retarded/causal Green functions. The negative radiation can be interpreted as an elementary absorption. Observe also that according to expression (23) the interaction energy density can be negative similarly to the electrostatic energy for two classical point charges of different signs.

The significance of individual EM fields in the new theory is manifested also in the existence of a group of gauge transformations in addition to the gauge transformation of the first or the second kind (known also as respectively global and local gauge transformation) $[12,19]$. Namely, we introduce a new gauge transformation

$$
A^{\ell \mu} \rightarrow A^{\ell \mu}+\partial^{\mu} \lambda^{\ell}(x), \quad \psi^{\ell} \rightarrow \mathrm{e}^{-\frac{\mathrm{i} q^{\ell}}{\chi \mathrm{c}} \lambda^{\ell}}(x) \psi^{\ell},
$$

where functions $\lambda^{\ell}(x), 1 \leq \ell \leq N$, are independent real-valued scalar functions of $x$ and

$$
\lambda_{\neq}^{\ell}=\sum_{\ell^{\prime} \neq \ell} \lambda^{\ell}
$$

A straightforward examination shows that for $N \geq 2$ the system Lagrangian $\mathcal{L}$ defined by (10)-(11) is invariant with respect to the gauge transformations (35). It is due to the gauge invariance with respect to elementary gauge transformations (35) the source current in the elementary Maxwell equations (16), (17) defined by (14) is exactly the conserved Noether's elementary current for every $\ell$. 


\section{Non-relativistic Dynamics of Localized Charges}

In this section, based on our relativistic model we introduce a non-relativistic model for the case where charges move slowly compared with speed of light. First we introduce field equations and the Lagrangian and briefly describe their derivation from the relativistic model.

Using frequency-shifting substitution $\psi=\mathrm{e}^{-\mathrm{i} \omega_{0} t} \psi_{\omega}^{\ell}(t, \mathbf{x})$ similar to (19) with $\psi_{\omega}^{\ell}$ which depends on both $t$ and $\mathbf{x}$ we obtain an obvious expression for the term $-\frac{1}{c^{2}} \tilde{\partial}_{t}^{\ell} \tilde{\partial}_{t}^{\ell} \psi^{\ell}-\kappa_{0}^{2} \psi^{\ell}$ in the Klein-Gordon equation (12). We neglect in this expression terms with the factor $\frac{1}{\mathrm{c}}$. Since magnetic fields of moving charges have coefficient $\frac{1}{c}$ and are small for small velocities, they are also neglected, and we retain only the external magnetic fields. Consequently, our non-relativistic approximation turns into the following nonlinear Schrödinger equation (NLS)

$$
\mathrm{i} \chi \partial_{t} \psi^{\ell}=-\frac{\chi^{2}}{2 m^{\ell}}\left(\tilde{\nabla}_{\mathrm{ex}}^{\ell}\right)^{2} \psi^{\ell}+q^{\ell}\left(\varphi_{\neq}^{\ell}+\varphi_{\mathrm{ex}}\right) \psi^{\ell}+\frac{\chi^{2}}{2 m^{\ell}}\left[G_{a}^{\ell}\right]^{\prime}\left(\left|\psi^{\ell}\right|^{2}\right) \psi^{\ell},
$$

where (i) $\psi^{\ell}=\psi_{\omega}^{\ell}$; (ii) $a$ is the size parameter; (iii) $\tilde{\nabla}_{\mathrm{ex}}^{\ell}=\nabla-\frac{\mathrm{i} q^{\ell}}{\chi c} \mathbf{A}_{\mathrm{ex}}$ is covariant gradient; (iv) $\varphi_{\neq}^{\ell}$ is defined by (6) and every potential $\varphi^{\ell}$ is determined from the equation

$$
\nabla^{2} \varphi^{\ell}=-4 \pi q^{\ell}\left|\psi^{\ell}\right|^{2}, \quad \ell=1, \ldots, N .
$$

We take then a particular solution of (37) given by Green's formula

$$
\varphi^{\ell}(t, \mathbf{x})=q^{\ell} \int_{\mathbb{R}^{3}} \frac{\left|\psi^{\ell}\right|^{2}(t, \mathbf{y})}{|\mathbf{y}-\mathbf{x}|} \mathrm{d} \mathbf{y} .
$$

Notice that in the nonrelativistic case the $\ell$-th b-charge is described by a pair $\left(\psi^{\ell}, \varphi^{\ell}\right)$ where the elementary EM field is represented only by the scalar electric potential $\varphi^{\ell}$. The above non-relativistic model has the following Lagrangian

$$
\begin{aligned}
\hat{\mathcal{L}}\left(\varphi,\left\{\psi^{\ell}\right\}_{\ell=1}^{N},\left\{\varphi^{\ell}\right\}_{\ell=1}^{N}\right)=\frac{1}{8 \pi}|\nabla \varphi|^{2}+\sum_{\ell} \hat{L}^{\ell}\left(\psi^{\ell}, \psi^{\ell *}, \varphi\right), \quad \text { where } \\
\hat{L}^{\ell}=\frac{\chi \chi^{\mathrm{i}}}{2}\left[\psi^{\ell *} \partial_{t} \psi^{\ell}-\psi^{\ell} \partial_{t} \psi^{\ell *}\right]-\frac{\chi^{2}}{2 m^{\ell}}\left\{\left|\tilde{\nabla}_{\mathrm{ex}}^{\ell} \psi^{\ell}\right|^{2}+G^{\ell}\left(\psi^{\ell *} \psi^{\ell}\right)\right\} \\
+G^{\ell}\left(\psi^{\ell *} \psi^{\ell}\right)-q^{\ell}\left(\varphi+\varphi_{\mathrm{ex}}-\varphi^{\ell}\right) \psi^{\ell} \psi^{\ell *}-\frac{1}{8 \pi}\left|\nabla \varphi^{\ell}\right|^{2},
\end{aligned}
$$

with $\psi^{\ell *}$ being the complex conjugate to $\psi^{\ell}$ and $\varphi=\sum_{\ell} \varphi^{\ell}$. Field equations (36), (37) coincide with the Euler-Lagrange equations derived from (39).

The properties and examples of the nonlinearities $G_{a}^{\ell}$ are provided in Sect. 3.1. As the result of charge conservation for solutions of (36) the norms $\left\|\psi^{\ell}\right\|^{2}$ remain constant and we impose the following charge normalization condition:

$$
\left\|\psi^{\ell}\right\|^{2}=\int_{\mathbb{R}^{3}}\left|\psi^{\ell}\right|^{2} \mathrm{~d} \mathbf{x}=1, \quad t \geq 0, \ell=1, \ldots, N .
$$


A motivation for this particular normalization is based on the formula (38) and the requirement that the elementary potential $\varphi^{\ell}$ is asymptotically the Coulomb's potential $q^{\ell} /|\mathbf{x}|$ for large $|\mathbf{x}|$.

According to the classical electrodynamics the evolution of a point charge $q$ of a mass $m$ and position vector $\mathbf{r}(t)$ in an external electromagnetic (EM) field in the non-relativistic case is governed by Newton's equation (4). In our model a charge is described by a wave function $\psi^{\ell}(t, \mathbf{x})$ governed by a system of nonlinear Schrodinger equations (36) coupled through corresponding electric potentials. We show below in regimes when wave functions $\psi^{\ell}(t, \mathbf{x})=\psi_{a}^{\ell}(t, \mathbf{x})$ remain well localized around their centers $\mathbf{r}_{\ell}(t)$ defined by

$$
\mathbf{r}^{\ell}(t)=\mathbf{r}_{a}^{\ell}(t)=\int_{\mathbb{R}^{3}} \mathbf{x}\left|\psi_{a}^{\ell}(t, \mathbf{x})\right|^{2} \mathrm{~d} \mathbf{x}
$$

that the centers satisfy approximately Newton's equations with Lorentz forces. The dependence of the nonlinearity of on the size parameter $a$ is defined by (21). We consider below two different regimes: (i) macroscopic dynamics, with a macroscopic spatial scale $R_{\text {macr }} \gg a$ and a bound state as in hydrogen atom. For macroscopic regimes we prove in Sect. 3.2 that when $a \rightarrow 0$ the centers of the interacting charges converge to solutions of the Newton's equations with the Lorentz forces if $\psi^{\ell}$ remain localized. We also provide examples of exact solutions in the form of accelerating solitons for which the localization assumption holds. For bound regimes we recover discrete frequency and energy spectrum matching the same for the Schrödinger hydrogen atom with any desired accuracy controlled by the ratio of Bohr radius and the size parameter $a$.

\subsection{Determination of Nonlinearity}

As we have already mentioned, the nonlinear self interaction function $G$ is determined from the charge equilibrium equation (20) based on the form factor (ground state) $\stackrel{\psi}{\psi}$. Important features of our nonlinearity include: (i) the boundedness or slow subcritical growth of its derivative $G^{\prime}(s)$ for $s \rightarrow \infty$ with consequent boundedness from below of the wave energy; (ii) slightly singular behavior about $s=0$, that is for small wave amplitudes. Throughout this section we have $\psi \geq 0$ and hence $|\psi|=\psi$. We introduce explicitly the dependence of the free ground state $\dot{\psi}$ on the size parameter $a>0$ as follows:

$$
\stackrel{\circ}{\psi}(r)=\stackrel{\circ}{\psi}_{a}(r)=a^{-3 / 2} \stackrel{\circ}{\psi}_{1}\left(a^{-1} r\right),
$$

and consequently $\stackrel{\leftrightarrow}{\psi}_{a}(r)$ satisfies the charge normalization condition (40) for every $a>0$. Definition (42) is consistent with (20) and (21). Let us take a look at the charge equilibrium equation (20) where the function ${ }_{\psi}(r)=\dot{\psi}_{a}(r)$ is assumed to be a smooth positive monotonically decreasing function of $r \geq 0$ and to satisfy the charge normalization condition of the form (40). Note that equations for a single resting charge without external field are obtained by setting $\partial_{t} \psi=0, \varphi_{\mathrm{ex}}=0, \mathbf{A}_{\mathrm{ex}}=0$ in (36). Obviously, the equations coincide with (20) and therefore the rest solutions of relativistic and non-relativistic equations coincide, as it should be expected in the case of zero velocity. 
Let us consider first the case $a=1$ and $\dot{\psi}_{a}=\dot{\psi}_{1}$. Since $\dot{\psi}_{1}^{2}(r)$ is a monotonic function, we can find its inverse $r=r\left(\psi^{2}\right)$ and (20) yields the following representation for $G^{\prime}\left(\stackrel{\circ}{\psi}_{1}^{2}\right)$ :

$$
G_{1}^{\prime}(s)=\frac{\nabla^{2} \dot{\psi}_{1}(r(s))}{\dot{\psi}_{1}(r(s))}, \quad 0=\dot{\psi}_{1}^{2}(\infty) \leq s \leq \dot{\psi}_{1}^{2}(0) .
$$

If we do not need $G^{\prime}(s)$ to be smooth, we extend $G^{\prime}(s)$ for $s \geq \dot{\psi}_{1}^{2}(0)$ as a constant, namely $G_{1}^{\prime}(s)=G_{1}^{\prime}\left(\dot{\psi}_{1}^{2}(0)\right)$ if $s \geq \dot{\psi}_{1}^{2}(0)$. The first derivative of such an extension at $s=\dot{\psi}_{1}^{2}(0)$ has a discontinuity point. If $\dot{\psi}_{a}(r)$ is a smooth function, we can define an extension of $G^{\prime}(s)$ for $s \geq \dot{\psi}_{1}^{2}(0)$ which is a smooth bounded function for all $r>0$, slowly growing functions $G^{\prime}(s)$ also can be used. For arbitrary $a>0$ we define $G_{a}^{\prime}(s)$ by formula (21) to be consistent with (42) and (43).

Example 1 The form factor $\dot{\psi}_{1}(r)$ decays as a power law: $\stackrel{\psi}{\psi}_{1}(r)=c_{\mathrm{pw}}\left(1+r^{2}\right)^{-p}$, $p>3 / 4$, where $c_{\mathrm{pw}}$ is the normalization factor. This function evidently is positive and monotonically decreasing. $G^{\prime}(s)$ is found then based on the relations (43). An elementary computation of $\nabla^{2} \stackrel{\circ}{\psi}_{1}$ shows that $G^{\prime}(s)$ has the form of a linear combination of power laws.

Example 2 Exponentially decaying form factor $\stackrel{\circ}{\psi}_{1}(r)=c_{\mathrm{e}} \mathrm{e}^{-\left(r^{2}+1\right)^{1 / 2}}$, where $c_{\mathrm{e}}$ is the normalization factor. An elementary computation based on (43) shows that for $s \leq c_{\mathrm{e}}^{2} \mathrm{e}^{-2}$

$$
G_{1}^{\prime}(s)=1-4 / \ln \left(c_{\mathrm{e}}^{2} / s\right)-4 / \ln ^{2}\left(c_{\mathrm{e}}^{2} / s\right)-8 / \ln ^{3}\left(c_{\mathrm{e}}^{2} / s\right) .
$$

We extend $G_{1}^{\prime}(s)$ for $s \geq c_{\mathrm{e}}^{2} \mathrm{e}^{-2}$ as a constant. To find $G_{a}^{\prime}(s)$ for arbitrary $a$ we use its representation (21).

Example 3 Gaussian form factor $\stackrel{\AA}{\psi}_{1}(r)=C_{g} \mathrm{e}^{-r^{2} / 2}$ with $C_{g}=\pi^{-3 / 4}$. Such a function is called gausson in [4]. Elementary computation based on (43) and (21) shows that for a given size parameter $a>0$ the nonlinearity, which we call logarithmic nonlinearity, is given by the formula

$$
G_{a}^{\prime}\left(|\psi|^{2}\right)=-a^{-2} \ln \left(a^{3}|\psi|^{2} / C_{g}^{2}\right)-3 a^{-2} .
$$

\subsection{Charges in Remote Interaction Regimes}

We show here that if the size parameter $a \rightarrow 0$ the dynamics of the centers of localized solutions is approximated by Newton's law of motion. This is done in the spirit of the well known in quantum mechanics Ehrenfest Theorem [15, 7, 23] and [4]. Wave-corpuscle solutions defined by (52), (53) provide an example of explicit localized solutions which have such a dynamics. As we stated, the Lagrangian $\hat{\mathcal{L}}$ in (39) is gauge invariant and every $\ell$-th charge has a 4-current $\left(\rho^{\ell}, \mathbf{J}^{\ell}\right)$ defined by

$$
\rho^{\ell}=q\left|\psi^{\ell}\right|^{2}, \quad \mathbf{J}^{\ell}=\left(\frac{\chi q^{\ell}}{m^{\ell}} \operatorname{Im} \frac{\nabla \psi^{\ell}}{\psi^{\ell}}-\frac{q^{\ell 2} \mathbf{A}_{\mathrm{ex}}}{m^{\ell} \mathrm{c}}\right)\left|\psi^{\ell}\right|^{2},
$$


which satisfies the continuity equations $\partial_{t} \rho^{\ell}+\nabla \cdot \mathbf{J}^{\ell}=0$ or explicitly

$$
\partial_{t}\left|\psi^{\ell}\right|^{2}+\nabla \cdot\left(\frac{\chi}{m^{\ell}} \operatorname{Im} \frac{\nabla \psi^{\ell}}{\psi^{\ell}}\left|\psi^{\ell}\right|^{2}-\frac{q^{\ell}}{m^{\ell} \mathrm{c}} \mathbf{A}_{\mathrm{ex}}\left|\psi^{\ell}\right|^{2}\right)=0 .
$$

Note that $\mathbf{J}^{\ell}$ defined by (45) agrees with the definition (14) of current $\mathbf{J}^{\ell}$ in Maxwell equations. Equations (46) can be obtained via multiplying (36) by $\psi^{\ell *}$ and taking imaginary part. Integrating the continuity equation we find that $\left\|\psi^{\ell}\right\|^{2}=$ const and we impose the normalization condition (40). Multiplying continuity equation (46) by $\mathbf{x}$ and integrating we find the following identities

$$
\frac{\mathrm{d} \mathbf{r}^{\ell}}{\mathrm{d} t}=\mathrm{v}^{\ell}, \quad \text { where } \mathrm{v}^{\ell}(t)=\int_{\mathbb{R}^{3}} \mathbf{v}^{\ell}(t, \mathbf{x}) \mathrm{d} \mathbf{x}, \mathbf{v}^{\ell}=\frac{1}{q^{\ell}} \mathbf{J}^{\ell} .
$$

Taking time derivative of (47) and using the field equations we obtain (see [3] for details) the following system for $\ell=1, \ldots, N$ :

$$
m^{\ell} \frac{\mathrm{d}^{2} \mathbf{r}^{\ell}(t)}{\mathrm{d}^{2} t}=q^{\ell} \int_{\mathbb{R}^{3}}\left[\left(\sum_{\ell^{\prime} \neq \ell} \mathbf{E}^{\ell^{\prime}}+\mathbf{E}_{\mathrm{ex}}\right)\left|\psi^{\ell}\right|^{2}+\frac{\mathbf{v}^{\ell} \times \mathbf{B}_{\mathrm{ex}}}{\mathrm{c}}\right] \mathrm{d} \mathbf{x},
$$

where $\mathbf{E}^{\ell^{\prime}}(t, \mathbf{x})=-\nabla \varphi^{\ell^{\prime}}(t, \mathbf{x}), \mathbf{E}_{\mathrm{ex}}$ and $\mathbf{B}_{\mathrm{ex}}$ are defined by (18).

We show below that (48) imply the following remarkable property: the positions $\mathbf{r}^{\ell}(t)$ satisfy with a high accuracy Newton's equations of motion for the system of $N$ point charges if the size parameter $a$ is small compared to the typical spatial scale $R_{\text {macr }}$ of variation of EM fields. More technical details and exact conditions needed for the derivation are provided in [3].

Suppose that for every $\ell$-th charge density $\left|\psi^{\ell}\right|^{2}$ and the corresponding current density $\mathbf{J}^{\ell}$ are localized in a small vicinity of the position $\mathbf{r}^{\ell}(t)$, this vicinity shrinks as $a \rightarrow 0$ and that $\left|\mathbf{r}^{\ell}(t)-\mathbf{r}^{\ell^{\prime}}(t)\right| \geq \gamma>0$ with $\gamma$ independent on $a$ on time interval $[0, T]$. Then if $a \rightarrow 0$ we get

$$
\left|\psi^{\ell}\right|^{2}(t, \mathbf{x}) \rightarrow \delta\left(\mathbf{x}-\mathbf{r}^{\ell}(t)\right), \quad \mathbf{v}^{\ell}(t, x) \rightarrow \mathbf{v}^{\ell}(t) \delta\left(\mathbf{x}-\mathbf{r}^{\ell}(t)\right),
$$

where the coefficients before the Dirac delta-functions are determined by the charge normalization conditions (40) and relations (47). Using potential representations (38) we infer from (49) the convergence of the potentials $\varphi^{\ell}$ to the corresponding Coulomb's potentials, namely

$$
\varphi^{\ell}(t, \mathbf{x}) \rightarrow \varphi_{\mathrm{C}}^{\ell}(t, \mathbf{x})=\frac{q^{\ell}}{\left|\mathbf{x}-\mathbf{r}^{\ell}\right|}, \quad-\nabla \varphi^{\ell}(t, \mathbf{x}) \rightarrow \mathbf{E}_{\mathrm{C}}^{\ell}(\mathbf{x})=q^{\ell} \frac{\mathbf{x}-\mathbf{r}^{\ell}}{\left|\mathbf{x}-\mathbf{r}^{\ell}\right|^{3}}
$$

as $a \rightarrow 0$. Hence, passing to the limit, we obtain the following system for $\ell=$ $1, \ldots, N$ :

$$
m^{\ell} \frac{\mathrm{d}^{2} \mathbf{r}^{\ell}}{\mathrm{d} t^{2}}=\sum_{\ell^{\prime} \neq \ell} q^{\ell} \mathbf{E}_{\mathrm{C}}^{\ell^{\prime}}\left(\mathbf{r}^{\ell}\right)+q^{\ell} \mathbf{E}_{\mathrm{ex}}\left(\mathbf{r}^{\ell}\right)+\frac{q^{\ell}}{\mathrm{c}} \mathbf{v}^{\ell} \times \mathbf{B}_{\mathrm{ex}}\left(\mathbf{r}^{\ell}\right) .
$$


The limit equations of motion are evidently Newton's equations of motion with the proper Lorentz forces. Note that in the derivation we essentially use the fact that the nonlinearity $G_{a}^{\prime}$, which, according to (21), singularly depends on $a$ as $a \rightarrow 0$, does not enter the system (48) explicitly.

In the case of a single charge the Lagrangian and the field equations are obtained by setting $N=1$ in (39), (36), (37). In this case evidently $\ell$ takes only one value and $\varphi_{\neq}^{\ell}=0$. For a special class of external fields we present now explicit solutions to the field equations (36), (37) for a single charge in the form of wave-corpuscles (accelerating solitons). We assume here for simplicity a purely electric external EM field, i.e. when $\mathbf{A}_{\mathrm{ex}}=0, \mathbf{E}_{\mathrm{ex}}(t, \mathbf{x})=-\nabla \varphi_{\mathrm{ex}}(t, \mathbf{x})$ (see [1,2] for a similar exact solution with non-zero magnetic field). We define then wave-corpuscle $\psi, \varphi$ by the following formula:

$$
\psi(t, \mathbf{x})=\mathrm{e}^{\mathrm{i} S / \chi} \dot{\psi}\left(\left|\mathbf{x}-\mathbf{r}^{\ell}(t)\right|\right), \quad S=m^{\ell} \mathbf{v}^{\ell}(t) \cdot\left(\mathbf{x}-\mathbf{r}^{\ell}(t)\right)+s_{\mathrm{p}}(t) .
$$

In the above formula $\Varangle$ is the form factor satisfying (20), $\varphi$ is determined by (38). Since $\dot{\psi}(|\mathbf{x}|)$ is center-symmetric, $\mathbf{r}^{\ell}$ satisfies (41).

Suppose that $\varphi_{\mathrm{ex}}(t, \mathbf{x})$ is a continuous function which is linear with respect to $\mathbf{x}$. Then $\psi$ defined by (52) provides an exact solution to (36), if $\mathbf{r}^{\ell}(t)$ and $\mathbf{v}^{\ell}(t)$ are determined from the equation

$$
m^{\ell} \frac{\mathrm{d}^{2} \mathbf{r}^{\ell}}{\mathrm{d} t^{2}}=q^{\ell} \mathbf{E}_{\mathrm{ex}}\left(t, \mathbf{r}^{\ell}\right), \quad \mathbf{v}^{\ell}=\frac{\mathrm{d} \mathbf{r}^{\ell}}{\mathrm{d} t},
$$

with an explicit formula for the phase shift $s_{\mathrm{p}}(t)$. The verification of the fact that an exact solution can be written in this form is straightforward and details can be found in [1], [2] or [3]. For the particular case of the logarithmic nonlinearity solutions of the form (52) were found in [4] in the form of accelerating gaussons.

The exponential factor in the wave-corpuscle solution of the form (52) can be identified with the de Broglie wave, for details see [1, 2]. Note that the construction of the solution (52) does not depend on a particular form of the nonlinearity $G^{\prime}=G_{a}^{\prime}$ as long as (20) is satisfied and the dependence on $a$ in (52) is only through $\dot{\psi}(\mid \mathbf{x}-$ $\mathbf{r} \mid)=a^{-3 / 2} \dot{\psi}_{1}\left(a^{-1}|\mathbf{x}-\mathbf{r}|\right)$. Obviously, if $\psi(t, \mathbf{x})$ is defined by (52) then $|\psi(t, \mathbf{x})|^{2} \rightarrow$ $\delta(\mathbf{x}-\mathbf{r})$ exactly as in (49).

\subsection{Hydrogen Atom Model}

Here we provide a sketch of our hydrogen atom (HA) model. In this model $\psi_{\ell}$ is obviously spinless though it is quite clear that extensions of this model to multicomponent $\psi_{\ell}$ is possible.

To model the hydrogen atom (HA) we set $N=2$ in the non-relativistic system (36), (37) where the indices $\ell$ take two values $\ell=1$, for electron, and $\ell=2$, for proton, and the charges values $q_{1}=-q=q_{2}$. The electric fields in the resting hydrogen atom have to be time-independent, hence $\left|\psi_{\ell}\right|^{2}$ in (37) must be time-independent too. Therefore we assume that only phase factors depend on time and consider the multi-harmonic solutions of this system, namely solutions of the form

$$
\psi^{\ell}(t, \mathbf{x})=\mathrm{e}^{-\mathrm{i} \omega_{\ell} t} \psi_{\ell}(\mathbf{x}), \quad \varphi^{\ell}(t, \mathbf{x})=\varphi_{\ell}(\mathbf{x}), \quad \ell=1,2 .
$$


Plugging the expressions (54) in (36), (37) we find that the functions $\psi_{\ell}(\mathbf{x})$ satisfy the following nonlinear eigenvalue problem

$$
\chi \omega_{\ell} \psi_{\ell}+\frac{\chi^{2}}{2 m^{\ell}} \nabla^{2} \psi_{\ell}-q_{\ell} \varphi_{\neq \ell} \psi_{\ell}=\frac{\chi^{2}}{2 m_{\ell}} G_{\ell}^{\prime}\left(\left|\psi_{\ell}\right|^{2}\right) \psi_{\ell},
$$

where, in accordance with (6), $\varphi_{\neq 1}=\varphi_{2}, \varphi_{\neq 2}=\varphi_{1}$ and $\varphi_{\ell}$ are defined by (38). We choose logarithmic nonlinearities $G_{\ell}^{\prime}=G_{\ell a}^{\prime}$ as in (44) implying that the frequencies $\omega_{1}, \omega_{2}$ and the values of particle energies satisfy Planck-Einstein formula $E=\hbar \omega$ exactly (see [3] for details and also [4] where a relation between the Planck-Einstein formula and the logarithmic nonlinearity was discovered in a different setting). We rewrite the system (55), as the following nonlinear eigenvalue problem

$$
\begin{aligned}
& \frac{\chi}{q^{2}} \omega_{1} \psi_{1}+\frac{a_{1}}{2} \nabla^{2} \psi_{1}+\frac{\varphi_{2}}{q_{2}} \psi_{1}=\frac{a_{1}}{2} G_{1}^{\prime}\left(\left|\psi_{1}\right|^{2}\right) \psi_{1}, \\
& \frac{\chi}{q^{2}} \omega_{2} \psi_{2}+\frac{a_{2}}{2} \nabla^{2} \psi_{2}+\frac{\varphi_{1}}{q_{1}} \psi_{2}=\frac{a_{2}}{2} G_{2}^{\prime}\left(\left|\psi_{2}\right|^{2}\right) \psi_{2},
\end{aligned}
$$

where $\varphi_{\ell}$ are defined by (38), $a_{\ell}=\frac{\chi^{2}}{q^{2} m_{\ell}}, \ell=1,2$. Note that the quantity $a_{1}$ turns into the Bohr radius if $\chi$ equals to the Planck constant $\hbar$, and $m_{1}, q$ are the electron mass and charge respectively. Here $\psi_{1}$ and $\psi_{2}$ are respectively the wave functions for the electron and the proton satisfying charge normalization condition

$$
\left\|\psi_{1}\right\|=1, \quad\left\|\psi_{2}\right\|=1 .
$$

The problem of finding frequencies $\omega_{1}, \omega_{2}$ can be reduced to finding critical values of the energy functional $\mathcal{E}\left(\psi_{1}, \psi_{2}\right)$ with the constraint (58) with $\omega_{1}, \omega_{2}$ being the Lagrange multipliers. An analysis of this variational problem (see [3] for details) shows that this problem can be reduced approximately to a simpler variational problem for energy $\mathcal{E}_{\mathrm{Cb}}\left(\Psi_{1}\right)$ of a single wave function in a way similar to the BornOppenheimer approximation in the quantum mechanics. The accuracy of approximation of lower energy levels of $\mathcal{E}\left(\psi_{1}, \psi_{2}\right)$ is controlled by the small parameter $b^{2} \ll 1$ where $b=\frac{m_{1}}{m_{2}}=\frac{a_{2}}{a_{1}} \simeq \frac{1}{1837}$ is the electron/proton mass ratio. Consequently, we obtain the following nonlinear eigenvalue problem for the electron wave function $\Psi_{1}$ and dimensionless spectral parameter $\omega$ :

$$
\omega \Psi_{1}+\frac{1}{2} \nabla^{2} \Psi_{1}+\frac{1}{|\mathbf{y}|} \Psi_{1}=\frac{1}{2} G_{1}^{\prime}\left(\left|\Psi_{1}\right|^{2}\right) \Psi_{1},
$$

subjected to the constraint $\left\|\Psi_{1}\right\|=1$.

We exploit the dependence of the nonlinearity $G_{1}^{\prime}=G_{1 a}^{\prime}$ on the parameter $\kappa=$ $a_{1} / a$ which is the ratio of the Bohr radius $a_{1}$ to the electron size parameter $a$. For small $\kappa$ the nonlinearity $G_{1}^{\prime}(s)=\kappa^{2} G_{11}^{\prime}\left(\kappa^{-3} s\right)$ is a small perturbation in the eigenvalue problem (59). Further detailed analysis of the corresponding variational problem shows that lower energy levels of the energy functional $\mathcal{E}_{\mathrm{Cb}}\left(\Psi_{1}\right)$ are arbitrary close to the energy levels of the linear Schrodinger operator for HA provided that $\kappa=a_{1} / a$ is sufficiently small. Consequently, based on estimates obtained in [3] we 
find that $n$-th lower frequency $\omega_{1 n}$ for solution of (56), (57) is given by the following approximate formula

$$
\chi \omega_{1 n}=-\frac{1}{n^{2}} \frac{q^{2}}{2 a_{1}}\left[1+O\left(b^{2}+\left(\frac{a_{1}}{a}\right)^{2}\left|\ln \left(\frac{a_{1}}{a}\right)\right|\right)\right], \quad n=1,2, \ldots
$$

The correction term $O\left(b^{2}+\left(a_{1} / a\right)^{2}\left|\ln \left(a_{1} / a\right)\right|\right)$ in (60) is small provided $b$ and $a_{1} / a$ are small. Observe that differences of energy levels of the nonlinear eigenvalue problem are very close to the same in the Rydberg formula with relative error of order $10^{-4}$ if $a_{1} / a$ is of order $10^{-2}$. Hence, if we assume that the size $a$ of a free electron is 100 times larger than the Bohr radius, then the introduced here hydrogen atom model provides a good quantitative agreement with the hydrogen spectroscopic data. We think that it is quite reasonable to assume that a free electron has a much larger size than an electron bound in a hydrogen atom where it is naturally contracted by the electric force of the positively charged proton.

We would like to point out that the provisional HA model from [2] based on a single EM field already provides for discrete energy levels, but as $a_{1} / a \rightarrow 0$ the limiting linear eigenvalue problem involves a potential $-q^{2} /|\mathbf{y}|+q \phi(\mathbf{y})$ where in addition to the Coulomb potential there is a term $q \phi(\mathbf{y})$ due to the electron EM selfinteraction. So, if there is EM self-interaction the limiting eigenvalue problem as $a_{1} / a \rightarrow 0$ does not turn into the same for the linear Schrodinger operator for the HA.

Now we briefly compare the above non-relativistic treatment of the HA with the treatment in the framework of the full relativistic version of our model. We start directly from the relativistic system in (12), (16), (17) and look for time-harmonic solutions with $\mathbf{A}^{\ell}=0$ and time-independent $\varphi^{\ell}$ using substitutions (19) and (54) as follows:

$$
\psi^{\ell}(t, \mathbf{x})=\mathrm{e}^{-\mathrm{i}\left(\omega_{\ell}+\omega_{0}\right) t} \psi_{\ell}(\mathbf{x}), \quad \varphi^{\ell}(t, \mathbf{x})=\varphi_{\ell}(\mathbf{x}),
$$

where $\ell=1,2, \omega_{0}=m \mathrm{c}^{2} / \chi=\mathrm{c} \kappa_{0}$. We arrive then at a system similar to (55)

$$
\left(m_{\ell} \mathrm{c}^{2}+\chi \omega_{\ell}-q^{\ell} \varphi_{\neq \ell}\right)^{2} \psi^{\ell}+\chi^{2} \mathrm{c}^{2} \nabla^{2} \psi_{\ell}-\chi^{2} \mathrm{c}^{2} G^{\ell \prime}\left(\psi_{\ell}^{*} \psi_{\ell}\right) \psi_{\ell}-m^{2} \mathrm{c}^{4} \psi_{\ell}=0 .
$$

Based on smallness of electron/proton mass ratio we similarly to the non-relativistic case arrive at the following eigenvalue problem for electron density which is a relativistic version of (59):

$$
\left(m_{1} \mathrm{c}^{2}+\chi \omega_{1}+q^{2} /|\mathbf{y}|\right)^{2} \psi_{1}+\mathrm{c}^{2} \chi^{2} \nabla^{2} \psi_{1}-\mathrm{c}^{2} \chi^{2} G^{\prime}\left(\left|\psi_{1}\right|^{2}\right) \psi_{1}-m_{1}^{2} \mathrm{c}^{4} \psi_{1}=0
$$

If the ratio $\kappa=a_{1} / a$ is small, the nonlinearity $G^{\prime}(s)=\kappa^{2} G^{\prime}\left(\kappa^{-3} s\right)$ can be treated as a small perturbation, and the linear part of (61) essentially determines the lower energy levels. Note that if we set $\chi=\hbar$ the linear part of (61) coincides with the relativistic version of the Schrodinger equation, [15, p. 309]. According to [15] the energy levels of this equation in a contrast to the non-relativistic hydrogen Schrodinger equation have a fine structure, the fine structure energy levels are given by Sommerfeld's formula and the relative scale of the fine structure is controlled by $\alpha^{2}$ where $\alpha$ is Sommerfeld's fine structure constant, $\alpha \simeq \frac{1}{137}$. This shows that in our relativistic 
model relativistic effects are present even in the case of zero velocities if the square of Sommerfeld's fine structure constant is not assumed to be negligible.

Note that in our treatment of charges at macroscopic scales in Sect. 3.2 we assume the electron size $a$ to be very small whereas in Sect. 3.3 we assume $\kappa=a_{1} / a$ to be very small. There is no contradiction in doing that if one takes into account the small value of the Bohr radius $a_{1} \sim 5.3 \times 10^{-11} \mathrm{~m}$ compared with the scale $R_{\text {macr }}$ of spatial variation of macroscopic external EM fields acting on the charge. The error of approximation of $\mathbf{r}^{\ell}(t)$ in (41) by the Newtonian trajectory found from (51) is of order $a^{2} / R_{\text {macr }}^{2} \ll 1$. In the treatment of the HA in this section we assume $\kappa^{2}=a_{1}^{2} / a^{2} \ll 1$. Taking $a \sim 10^{2} a_{1}$ we arrive at the restriction $R_{\text {macr }} \gg 5.3 \times 10^{-9} \mathrm{~m}$ which is an estimate of the spatial scale at which external EM fields can vary and the motion of charges caused by the fields can be described by Newton's equations with the Lorentz forces with a good accuracy.

Acknowledgement The research was supported through Dr. A. Nachman of the U.S. Air Force Office of Scientific Research (AFOSR), under grant number FA9550-04-1-0359.

Open Access This article is distributed under the terms of the Creative Commons Attribution Noncommercial License which permits any noncommercial use, distribution, and reproduction in any medium, provided the original author(s) and source are credited.

\section{References}

1. Babin, A., Figotin, A.: Wave-corpuscle mechanics for electric charges. J. Stat. Phys. 138, 912-954 (2010)

2. Babin, A., Figotin, A.: Wave-corpuscle mechanics for elementary charges. e-print available online at arXiv:0812.2686

3. Babin, A., Figotin, A.: Some mathematical problems in a neoclassical theory of electric charges. Discrete Contin. Dyn. Syst. A 27(4), 1283-1326 (2010)

4. Bialynicki-Birula, I., Mycielski, J.: Nonlinear wave mechanics. Ann. Phys. 100, 62-93 (1976)

5. Corduneanu, C.: Almost Periodic Oscillations and Waves. Springer, Berlin (2009)

6. Einstein, A.: Über die Entwicklung unserer Anschauungen über das Wesen und die Konstitution der Strahlung. Phys. Z., vol. 10 (1909). English translation: On the development of our view concerning the nature and constitution of radiation. In: The Collected Papers, vol. 2, The Swiss Years Writings 1900-1909, Princeton (1989)

7. Goldstein, H., Poole, C., Safko, J.: Classical Mechanics, 3rd edn. Addison-Wesley, Reading (2000)

8. Griffiths, D.: Introduction to Electrodynamics, 3rd edn. Prentice Hall, New York (1999)

9. Jackson, J.: Classical Electrodynamics, 3rd edn. Wiley, New York (1999)

10. Kiessling, M.: Quantum Abraham models with de Broglie-Bohm laws of quantum motion. e-print available online at arXiv:physics/0604069v2

11. Mead, C.: Collective Electrodynamics-Quantum Foundations of Electromagnetism. MIT Press, Cambridge (2000)

12. Pauli, W.: Relativistic field theories of elementary particles. Rev. Mod. Phys. 13, 203-234 (1941)

13. Pearle, P.: Classical Electron Models. In: Teplitz, D. (ed.) Electromagnetism Paths to Research, pp. 211-295. Plenum, New York (1982)

14. Rohrlich, F.: Classical Charged Particles, 3rd edn. World Scientific, Singapore (2007)

15. Schiff, L.: Quantum Mechanics. McGraw-Hill, New York (1949)

16. Schwinger, J.: Electromagnetic mass revisited. Found. Phys. 13(3), 373-383 (1983)

17. Spohn, H.: Dynamics of Charged Particles and Their Radiation Field. Cambridge University Press, Cambridge (2004)

18. van Bladel, J.: Electromagnetic Fields, 2nd edn. IEEE Press, New York (2007)

19. Wentzel, G.: Quantum Theory of Fields. Dover, New York (2003) 
20. Wheeler, J., Feynman, R.: Interaction with the absorber as the mechanism of radiation. Rev. Mod. Phys. 17(2-3), 157-181 (1945)

21. Wheeler, J., Feynman, R.: Classical electrodynamics in terms of direct interparticle action. Rev. Mod. Phys. 21(3), 425-433 (1949)

22. Yaghjian, A.: Relativistic Dynamics of a Charged Sphere: Updating the Lorentz-Abraham Model, 2nd edn. Springer, Berlin (2006)

23. Zeh, H.: The Physical Basis of the Direction of Time, 5th edn. Springer, Berlin (2007) 